\title{
q-Truncation Error associated with Numerical Differentiation
}

\author{
Prashant Singh ${ }^{1}$, Pramod Kumar Mishra ${ }^{2}$ \\ ${ }^{1}$ (Department of Computer Science, Banaras Hindu University, India) \\ ${ }_{2}^{2}$ (Deparment of Computer Science, Banaras Hindu University, India)
}

\begin{abstract}
This paper is devoted to derivation of q-analogues of Truncation Errors associated with numerical differentiation. To analyse the error in numerical differentiation Taylor polynomial with remainders are useful and we have used different approximations to calculate errors. It also deals with comparison of accuracy of $q$ analogue of Taylor's Series using both single and double q parameters and Truncation Errors related to it.
\end{abstract}

Keywords: Basic, q-special function, q-Truncation Error, basic analogue, T.E.(Truncation Error)

\section{Introduction}

A q-hypergeometric function is an analogue of an ordinary hypergeometric function with addition of an extra parameter q, where $0<\mathrm{q}<1$. When $\mathrm{q}$ tends to one from left, the q hyper geometric function tends to normal hyper geometric function.

\section{1 q-Number System}

$[\alpha ; q]=\frac{1-q^{\alpha}}{1-q}=1+q+q^{2}+\ldots+q^{(\alpha-1)}$

\section{2 q-Factorial Notation}

$[\mathrm{n} ; \mathrm{q}] !=[\mathrm{n} ; \mathrm{q}][\mathrm{n}-1 ; \mathrm{q}] \ldots[3 ; \mathrm{q}][2 ; \mathrm{q}][1 ; \mathrm{q}]$

\section{3 q-exponential function}

$\mathrm{E}_{\mathrm{q}}(\mathrm{x})=\sum_{r=0}^{\infty} x^{r} /[r ; q]$ !

$\mathrm{E}_{\mathrm{q}}^{-1}(\mathrm{x})=\sum_{r=0}^{\infty} x^{r} q^{\frac{r(r-1)}{2}} /[\mathrm{r} ; \mathrm{q}]$ !

$\mathrm{E}(\mathrm{q}, \mathrm{x})=\sum_{r=0}^{\infty} x^{r} q^{\frac{r(r-1)}{4}} /[\mathrm{r} ; \mathrm{q}]$ !

A whole family of q exponential function can be defined as

$\mathrm{E}(\mathrm{q}, \beta ; \mathrm{x})=\sum_{r=0}^{\infty} x^{r} q^{\beta \mathrm{r}(\mathrm{r}-1)} /[\mathrm{r} ; \mathrm{q}]$ !

depending upon value of $\beta$ i.e. $\beta=0, \beta=1 / 2$ and $\beta=1 / 4$ repectively.

Unlike ordinary exponential function $\mathrm{E}_{\mathrm{q}}(\mathrm{x})$ has a finite radius of convergence if $|\mathrm{q}|<1$. It converges uniformly with respect to $\mathrm{x}$ if $|\mathrm{x}|<(1-\mathrm{q})^{-1}$ and diverges if

$|x|>(1-q)^{-1}$

If $|\mathrm{q}|>1$, we have convergence for all values of $\mathrm{x}$.

$\mathrm{E}_{\mathrm{q}}(\mathrm{x})=\prod_{r=0}^{\infty}\left(1+x\left(1-q^{-1}\right) q^{-r}\right)$

$\mathrm{E}_{1 / \mathrm{q}}(\mathrm{x})=\prod_{r=0}^{\infty}\left(1+x(1-q) q^{r}\right)$

\section{4 q-Circular Function}

$\sin _{q}(\mathrm{x})=\left\{\mathrm{aE}_{\mathrm{q}}(\mathrm{ix})+\mathrm{E}_{\mathrm{q}}(-\mathrm{ix})\right\} / 2 \mathrm{i}=\mathrm{x}_{0} \mathrm{~F}_{1}\left(-; 3 / 2 ; \mathrm{q}^{2} ;-\left[1 / 2 ; \mathrm{q}^{2}\right]^{2} \mathrm{x}^{2}\right)$

$\cos _{\mathrm{q}}(\mathrm{x})=\left\{\mathrm{E}_{\mathrm{q}}(\mathrm{ix})+\mathrm{E}_{\mathrm{q}}(-\mathrm{ix})\right\} / 2={ }_{0} \mathrm{~F}_{1}\left(-; 1 / 2 ; \mathrm{q}^{2} ;\left[1 / 2 ; \mathrm{q}^{2}\right]^{2} \mathrm{x}^{2}\right)$

Both $\sin _{q}(\mathrm{x})$ and $\cos _{\mathrm{q}}(\mathrm{x})$ converges for $|\mathrm{x}|>(1-\mathrm{q})^{-1}$, while if $|\mathrm{q}|>=1$, series converges for finite value of $\mathrm{x}$.

\section{5 q- Differentiation operator}

$D_{\mathrm{q}, \mathrm{x}} \mathrm{f}(\mathrm{x})=\frac{\mathrm{f}(\mathrm{qx})-\mathrm{f}(\mathrm{x})}{x(q-1)}$

Formula for q-differentiation by parts

$D_{q, x}(f(x) g(x))=g(x) D_{q, x} f(x)+f(x) D_{q, x} g(x)$

$\mathrm{D}_{\mathrm{q}, \mathrm{x}} \frac{f(\mathrm{x})}{g(x)}=\frac{\mathrm{g}(\mathrm{x}) \mathrm{Dq}, \mathrm{x} f(\mathrm{x})-\mathrm{f}(\mathrm{x}) \mathrm{Dq}, \mathrm{x}(\mathrm{x})}{\mathrm{g}(\mathrm{x}) \mathrm{g}(\mathrm{qx})}$ 
1.6 Ward-Alsalam [2] q-differentiation operator containing two parameters

$D_{q_{1} q_{2}} \mathrm{f}(\mathrm{x})=\frac{f\left(q_{1} x\right)-f\left(q_{2} x\right)}{x\left(q_{1}-q_{2}\right)}$ where $\mathrm{q}_{1}=\mathrm{q}_{2}{ }^{-1}$

$D D_{q_{1} q_{2}} f(x)=\frac{\left[q_{2} f\left(q_{1}^{2} x\right)+q_{1} f\left(q_{2}^{2} x\right)-q_{1} \mathrm{f}\left(q_{1} q_{2} x\right)-q_{2} \mathrm{f}\left(q_{1} q_{2} x\right)\right]}{\left(q_{1}-q_{2}\right)^{2} x^{2}}$

\section{7 q-Taylor Series}

Jackson(1909) introduced q analogue of Taylor series

$f(x)=f(a)+\frac{(x-a)^{(1)}}{[1: q)} D_{q} f(a)+\frac{(x-a)^{(2)}}{[2 ; q]} D_{q}^{2} f(a)+\ldots+\frac{(x-a)^{(n)}}{[n ; q]} D_{q}^{n} \mathrm{f}(\mathrm{a})$

, where $\mathrm{R}_{\mathrm{n}}=\frac{(x-a)(n+1)}{[n+1 ; q]} D^{(n+1)} \mathrm{f}(\xi)$, where $\xi$ lies between $x$ and $a$.

It can be rewritten as

$f(x)=\sum_{n=0}^{\infty} \frac{(1-q)^{n}}{(q ; q)_{n}} D_{q}^{n} f(a)[x-a]_{n}$

For double parameter it can be written as

$f(x)=f(a)+\frac{(x-a)^{(1)}}{[1: q)} D_{q_{1} q_{2}} f(a)+\frac{(x-a)^{(2)}}{[2 ; q]} D_{q_{1} q_{2}}^{2} f(a)+\ldots+\frac{(x-a)^{(n)}}{[n ; q]} D_{q_{1} q_{2}}^{n} \mathrm{f}(\mathrm{a})$

where $\mathrm{q}_{1}$ and $\mathrm{q}_{2}$ are inverses of each other.

\section{Error Associated with q-analogue of Taylor Series}

Whenever we use approximations, it is important to try and keep track of the error, if possible. To analyse the error in the approximation we do Taylor expansion of $\mathrm{f}(\mathrm{a}+\mathrm{h})$. If we rearrange Taylor Series Expansion of $\mathrm{f}(\mathrm{a}+\mathrm{h})$. We get $f^{\prime}(a)-\frac{f(a+h)-f(a)}{h}=\frac{-h}{2} f^{\prime \prime}\left(\xi_{h}\right)$

This is referred to as Truncation Error. It would be better to get rid of $\xi_{h}$ and for that we take absolute value of both sides and in order to minimize this error we can approximate $f^{\prime \prime}(a)$ instead of $f^{\prime}(a)$.We can also approximate $f^{\prime}(a)$ by $\frac{\mathrm{f}(\mathrm{a}+\mathrm{h})-\mathrm{f}(\mathrm{a}-\mathrm{h})}{2 \mathrm{~h}}$ and calculate Truncation Error as well. But our focus is to compare qanalogue of Truncation Error with classical methods of calculating error.

Let us take a problem

Taylor series approximation of $\mathrm{e}^{\mathrm{x}}$ about $\mathrm{x}=0.1$ (also we can write it as $\operatorname{Exp}(0.1)$ )

Taking first five terms in the Taylor Seires of $\mathrm{e}^{\mathrm{x}}$ for $\mathrm{x}=0.1$ gives 1.105170833

Correct value of $\mathrm{e}^{\mathrm{x}}$ (for $\left.\mathrm{x}=0.1\right)=1.105170918$

Error $=$ Correct value-calculated value (upto 5 terms $)=\operatorname{Exp}(0.1)-\operatorname{Exp}(0.1)$ till five terms $=8.47423 \mathrm{E}-08$

Table 1: Table for $\mathrm{E}_{\mathrm{q}}(\mathrm{x})$ for different values of $\mathrm{q}$ at $\mathrm{n}=4$ (till five terms)

\begin{tabular}{|c|c|}
\hline Values of $q$ & $\mathbf{E}_{\mathrm{q}}(\mathbf{x})$ \\
\hline 0.9999999 & $\mathbf{1 . 1 0 5 1 7 0 8 3 4}$ \\
\hline 0.999 & $\mathbf{1 . 1 0 5 1 7 3 5 9 7}$ \\
\hline 0.99 & 1.10519861 \\
\hline
\end{tabular}

Conclusion: $\operatorname{Exp}(0.1)\left(\right.$ Correct Value of $\left.\mathrm{e}^{\mathrm{x}}\right)-\mathrm{E}_{\mathrm{q}=0.9999999, \mathrm{n}=4}(\mathrm{x})=8.44661 \mathrm{E}-08$ which is lesser than the error which we have calculated above by classical method.

\section{Theorem 3.1}

\section{III. q-Truncation Error associated with numerical differentiation}

Suppose $f$ and its first two derivatives are continuos near a and suppose we approximate $D_{q} f(a)$ by $\frac{f(a q)-f(a)}{a(q-1)}$. The truncation error in this approximation is bounded by

$\left|D_{q} f(a)-\frac{f(a q)-f(a)}{a(q-1)}\right| \leq \frac{a(q-1)}{(1+q)}\left|D_{q}^{2} f(\xi)\right|$

\section{Theorem 3.2}

Suppose $\mathrm{f}$ and its first two derivatives are continuos near a and suppose we approximate $D_{q_{1} q_{2}} f(a)$ by $\frac{f\left(a q_{1}\right)-f\left(a q_{2}\right)}{a\left(q_{1}-q_{2}\right)}$. The truncation error in this approximation is bounded by

$\left|D_{q_{1} q_{2}} f(a)-\frac{f\left(a q_{1}\right)-f\left(a q_{2}\right)}{a\left(q_{1}-q_{2}\right)}\right| \leq \frac{a\left(q_{1}-q_{2}\right)}{(1+q)}\left|D_{q_{1} q_{2}}^{2} f(a)\right|$ 
3.1 Truncation Error associated with classical method

Let us take a problem $f(x)=\operatorname{Exp}(0.1)$ for different values of $h$.

Table 2: Truncation Error by classical method

\begin{tabular}{|c|c|c|}
\hline value of $\mathbf{h}$ & $\mathbf{h} / 2$ & TRUNCATION ERROR \\
\hline 0.1 & 0.05 & 0.055258546 \\
\hline 0.01 & 0.005 & 0.005525855 \\
\hline 0.001 & 0.0005 & 0.000552585 \\
\hline 0.0001 & 0.00005 & $5.52585 \mathrm{E}-05$ \\
\hline 0.00001 & 0.000005 & $5.52585 \mathrm{E}-06$ \\
\hline 0.5 & 0.25 & 0.27629273 \\
\hline
\end{tabular}

3.2 Calculation of Truncation Error by q method using Theorem 3.1

Table 3: Truncation Error by q method

\begin{tabular}{|c|c|c|}
\hline VALUE OF q & $D_{q} D_{q} \operatorname{EXP}(0.1)$ & TRUNCATION ERROR \\
\hline 0.999 & 1.104507921 & $-5.5253 \mathrm{E}-05$ \\
\hline 0.98 & 1.091948073 & -0.001102978 \\
\hline 0.97 & 1.085365818 & -0.001652841 \\
\hline 0.96 & 1.078802799 & -0.002201638 \\
\hline 0.95 & 1.072258859 & -0.002749382 \\
\hline 0.94 & 1.065733843 & -0.003296084 \\
\hline 0.93 & $\mathbf{1 . 0 5 9 2 2 7 5 9 7}$ & -0.003841758 \\
\hline 0.92 & 1.052739966 & -0.004386417 \\
\hline 0.91 & 1.046270799 & -0.004930072 \\
\hline 0.9 & 1.039819944 & -0.005472737 \\
\hline 0.89 & 1.033387248 & -0.006014423 \\
\hline 0.88 & 1.026972563 & -0.006555144 \\
\hline 0.87 & 1.020575738 & -0.007094912 \\
\hline 0.86 & 1.014196624 & -0.007633738 \\
\hline 0.85 & 1.007835074 & -0.008171636 \\
\hline 0.84 & 1.00149094 & -0.008708617 \\
\hline $\mathbf{0 . 8 3}$ & 0.995164076 & -0.009244694 \\
\hline 0.82 & 0.988854335 & -0.009779878 \\
\hline 0.81 & 0.982561574 & -0.010314182 \\
\hline 0.8 & 0.976285647 & -0.010847618 \\
\hline 0.79 & 0.970026412 & -0.011380198 \\
\hline 0.78 & 0.963783724 & -0.011911934 \\
\hline 0.72 & 0.926667201 & -0.01508528 \\
\hline 0.71 & 0.92053641 & -0.015611436 \\
\hline
\end{tabular}

3.3Truncation Error associated with q method with two parameters using Theorem 3.2

Table 3:Truncation Error by using two parameters using Theorem 3.2 for different values of q1

\begin{tabular}{|l|l|l|l|l|l|l|}
\hline & & & & & & \\
$q_{1}$ & $q_{2}$ & $\operatorname{EXP}\left(q_{1} 0.1\right)$ & $\operatorname{EXP}\left(q_{2} 0.1\right)$ & $\operatorname{Dq}_{1} q_{2} f(x)$ & DDquncation \\
\hline 0.999 & 1.001 & 1.1050604 & 1.1052816 & 1.105171 & 1.105171609 & $-5.53 E-05$ \\
\hline 0.99 & 1.0101 & 1.1040663 & 1.1062878 & 1.1051767 & 1.105241993 & -0.000555 \\
\hline 0.98 & 1.02041 & 1.1029628 & 1.1074287 & 1.1051942 & 1.105458134 & -0.001117 \\
\hline 0.97 & 1.03093 & 1.1018604 & 1.1085943 & 1.1052239 & 1.105823877 & -0.001684 \\
\hline 0.96 & 1.04167 & 1.1007591 & 1.1097854 & 1.1052661 & 1.106343985 & -0.002258 \\
\hline
\end{tabular}




\begin{tabular}{|c|c|c|c|c|c|c|}
\hline 0.95 & 1.05263 & 1.0996589 & 1.1110029 & 1.1053212 & 1.107023455 & -0.002839 \\
\hline 0.94 & 1.06383 & $\mathbf{1 . 0 9 8 5 5 9 7}$ & $\mathbf{1 . 1 1 2 2 4 7 8}$ & 1.1053896 & 1.107867542 & -0.003426 \\
\hline 0.93 & 1.07527 & 1.0974617 & 1.1135208 & 1.1054718 & 1.108881768 & -0.004022 \\
\hline 0.92 & 1.08696 & 1.0963648 & 1.114823 & 1.1055682 & 1.11007194 & -0.004625 \\
\hline 0.91 & 1.0989 & 1.095269 & 1.1161554 & 1.1056793 & 1.111444173 & $-\mathbf{0 . 0 0 5 2 3 7}$ \\
\hline 0.9 & 1.11111 & 1.0941743 & 1.1175191 & 1.1058056 & 1.113004906 & -0.005858 \\
\hline 0.89 & 1.1236 & 1.0930807 & 1.1189151 & 1.1059476 & 1.114760924 & -0.006488 \\
\hline 0.88 & 1.13636 & 1.0919881 & 1.1203447 & 1.1061058 & 1.116719383 & -0.007128 \\
\hline 0.87 & 1.14943 & 1.0908967 & 1.121809 & 1.1062808 & 1.118887836 & $\underline{-0.007778}$ \\
\hline 0.86 & 1.16279 & 1.0898063 & 1.1233093 & 1.1064733 & 1.121274257 & -0.00844 \\
\hline 0.84 & 1.19048 & 1.0876289 & 1.1264236 & 1.1069129 & 1.126735204 & $\begin{array}{r}-0.009798 \\
\end{array}$ \\
\hline 0.83 & 1.20482 & 1.0865418 & 1.1280404 & 1.1071615 & 1.129828079 & -0.010496 \\
\hline 0.82 & 1.21951 & 1.0854558 & 1.129699 & 1.1074301 & 1.133175696 & -0.011207 \\
\hline 0.81 & 1.23457 & 1.0843709 & 1.1314011 & 1.1077196 & 1.136788651 & -0.011933 \\
\hline 0.8 & 1.25 & 1.0832871 & 1.1331485 & 1.1080308 & 1.140678189 & -0.012674 \\
\hline 0.74 & 1.35135 & 1.0768068 & 1.1446915 & 1.1104033 & 1.170548795 & -0.017491 \\
\hline 0.73 & 1.36986 & 1.0757305 & 1.1468124 & 1.1108925 & 1.176755613 & -0.018366 \\
\hline 0.71 & 1.40845 & 1.0735812 & 1.1512463 & 1.1119617 & 1.190380532 & $\begin{array}{r}-0.020188 \\
\end{array}$ \\
\hline 0.7 & 1.42857 & 1.0725082 & 1.153565 & 1.1125445 & 1.197840196 & -0.021138 \\
\hline 0.69 & 1.44928 & 1.0714362 & $\mathbf{1 . 1 5 5 9 5 5 8}$ & 1.1131613 & 1.205761595 & -0.022118 \\
\hline 0.68 & 1.47059 & 1.0703653 & 1.1584221 & $\mathbf{1 . 1 1 3 8 1 3 6}$ & 1.214169561 & -0.023127 \\
\hline 0.67 & 1.49254 & 1.0692955 & 1.1609675 & 1.1145032 & 1.22309083 & -0.024169 \\
\hline 0.63 & 1.5873 & 1.0650268 & 1.1720216 & 1.1176708 & 1.264521526 & $-\mathbf{- 0 . 0 2 8 7 0 4}$ \\
\hline 0.62 & 1.6129 & 1.0639623 & 1.1750261 & $\mathbf{1 . 1 1 8 5 7 5 4}$ & 1.276491541 & -0.029942 \\
\hline 0.54 & 1.85185 & 1.0554846 & 1.2034413 & 1.1278459 & 1.402932839 & -0.041906 \\
\hline 0.53 & 1.88679 & 1.0544296 & 1.2076535 & 1.1293097 & 1.423556355 & -0.04373 \\
\hline 0.52 & 1.92308 & $\mathbf{1 . 0 5 3 3 7 5 7}$ & 1.2120434 & 1.130855 & 1.445534495 & -0.045648 \\
\hline 0.51 & 1.96078 & 1.0523229 & 1.2166223 & 1.1324869 & 1.468978659 & -0.047669 \\
\hline 0.5 & 2 & 1.0512711 & 1.2214028 & 1.1342111 & 1.494013097 & -0.0498 \\
\hline
\end{tabular}

Conclusion: Truncation Error for $\mathrm{q}$ method for single as well as double parameter is lesser than classical method.for value of q near to one.

3.3 Truncation Error associated with q method considering three derivatives of $f$ and ignoring higher order derivatives

Theorem 3.3

Suppose $\mathrm{f}$ and its first three derivatives are continuous near a and suppose we approximate $D_{q}^{2} f(a)$ by $\frac{\left(f\left(q^{2} a\right)-[2 ; q] f(q a)+q f(a)\right)}{q a^{2}(1-q)^{2}}$. The truncation error in this approximation is bounded by

$\left|D_{q}^{2} f(a)-\frac{\left(f\left(q^{2} a\right)-[2 ; q] f(q a)+q f(a)\right)}{q a^{2}(1-q)^{2}}\right| \leq \frac{a^{2}(q-1)^{2}}{4[3 ; q] !}\left|D_{q}^{3}\left(f\left(\xi_{1}\right)+f\left(\xi_{2}\right)\right)\right| \leq \frac{a^{2}(q-1)^{2}}{2[3 ; q]}\left|D_{q}^{3} f(\xi)\right|$

, where $\mathrm{f}(\xi)$ is maximum among $\mathrm{f}\left(\xi_{1}\right)$ and $\mathrm{f}\left(\xi_{2}\right)$

Theorem 3.4 
Suppose $\mathrm{f}$ and its first three derivatives are continuous near a and suppose we approximate $D_{q}^{2} f(a)$ by $\frac{\left[q_{2} f\left(q_{1}^{2} x\right)+q_{1} f\left(q_{2}^{2} x\right)-q_{1} \mathrm{f}\left(q_{1} q_{2} x\right)-q_{2} \mathrm{f}\left(q_{1} q_{2} x\right)\right]}{\left(q_{1}-q_{2}\right)^{2} x^{2}}$, then Truncation Error is bounded by

$\left|D D_{q 1, q 2} f(a)-\frac{q_{2} f\left(q_{1}^{2} x\right)+q_{1} f\left(q_{2}^{2} x\right)-q_{1} \mathrm{f}\left(q_{1} q_{2} x\right)-q_{2} \mathrm{f}\left(q_{1} q_{2} x\right)}{\left(q_{1}-q_{2}\right)^{2} x^{2}}\right| \leq \frac{a^{2}\left(q_{1}-q_{2}\right)^{2}}{4[3 ; q] !}\left|D_{q 1, q 2}^{3}\left(f\left(\xi_{1}\right)+f\left(\xi_{2}\right)\right)\right| \leq$ $\frac{a^{2}\left(q_{1}-q_{2}\right)^{2}}{4[3 ; q] !}\left|D_{q 1, q 2}^{3} f(\xi)\right|$,

where $f(\xi)$ is maximum among $f\left(\xi_{1}\right)$ and $f\left(\xi_{2}\right)$

3.4 Calculation of Truncation Error by classical methods and its q-analogue using single and double parameters by approximating second order derivative of $f(x)$.

Let us see same problem $f(x)=e^{x}$ and calculate truncation error considering three derivatives of $f$ applying Theorem 3.3 and Theorem 3.4

Table 4: Truncation error by applying classical methods by approximating second derivative of $\mathrm{f}$.

\begin{tabular}{|l|l|l|}
\hline$h$ & $h^{2} / 6$ & T.E. \\
\hline 0.5 & 0.04166667 & 0.046048788 \\
\hline 0.1 & 0.00166667 & 0.001841952 \\
\hline 0.01 & $1.6667 \mathrm{E}-05$ & $1.84195 \mathrm{E}-05$ \\
\hline 0.001 & $1.6667 \mathrm{E}-07$ & $1.84195 \mathrm{E}-07$ \\
\hline 0.0001 & $1.6667 \mathrm{E}-09$ & $1.84195 \mathrm{E}-09$ \\
\hline
\end{tabular}

Table 5:Truncation Error by applying Theorem3.3 using single parameter q

\begin{tabular}{|l|l|}
\hline$q$ & TE \\
\hline 0.999 & $1.84472 E-09$ \\
\hline 0.99 & $1.86984 E-07$ \\
\hline 0.98 & $7.59308 E-07$ \\
\hline 0.97 & $1.73452 E-06$ \\
\hline 0.96 & $3.13083 E-06$ \\
\hline 0.8 & 0.000100653 \\
\hline 0.7 & 0.000267165 \\
\hline 0.6 & 0.000563863 \\
\hline 0.5 & 0.001052544 \\
\hline
\end{tabular}

Table 6: Truncation error using two parameters

\begin{tabular}{|l|l|l|}
\hline $\mathrm{q}_{1}$ & $\mathrm{q}_{2}$ & $\mathrm{TE}$ \\
\hline 0.999 & 1.001001 & $1.84656 \mathrm{E}-09$ \\
\hline 0.99 & 1.01010101 & $1.88878 \mathrm{E}-07$ \\
\hline 0.98 & 1.02040816 & $7.74883 \mathrm{E}-07$ \\
\hline 0.97 & 1.03092784 & $1.78858 \mathrm{E}-06$ \\
\hline 0.96 & 1.04166667 & $3.26264 \mathrm{E}-06$ \\
\hline 0.8 & 1.25 & 0.000127389 \\
\hline 0.7 & 1.42857143 & 0.000393931 \\
\hline 0.6 & 1.66666667 & 0.001002423 \\
\hline 0.5 & 2 & 0.002368223 \\
\hline
\end{tabular}

Conclusion: Truncation error associated with numerical differentiation considering three derivatives of $f$ also gives accurate result at $\mathrm{q}=0.999$.

\section{Calculation}

We have done all calculation by $\mathrm{C}++$ Programming language and verified it by statistical calculator. 


\section{Conclusions}

q method of calculating Truncation Error can minimize error more efficiently if we choose value of q near to one. q method can also be applied to numerical integration for reducing errors and it helps result to converge fast in comparison with classical methods.

\section{References:}

[1] G. Gasper and M. Rahman, Basic Hypergeometric Series, CambridgeUniversity Press, Cambridge, 1990.

[2] Thomas Ernst, A method for q-Calculus, Journal of Nonlinear Mathematical Physics, 2003

[3] Prashant Singh, Pramod Kumar Mishra and R.S.Pathak ,q-Iterative Methods, IOSR Journal of Mathematics (IOSR-JM), Volume 8, Issue 6 (Nov. - Dec. 2013), PP 01-06 